Abstract S110 Table 1 Effect of PR in COPD vs. ILD

\begin{tabular}{|c|c|c|c|}
\hline Outcome Measure (SD) & COPD & ILD & $\begin{array}{l}\text { p-value (two tailed) } \\
\text { Difference between groups }\end{array}$ \\
\hline$\Delta \mathrm{ISWT}$ metres $(n=46)$ & $49.4(59.6)$ & $27.4(58.8)$ & ns \\
\hline$\Delta$ ESWT seconds $(n=30)$ & $464(360)$ & 365 (342) & ns \\
\hline \multicolumn{4}{|l|}{$\operatorname{HAD}(n=51)$} \\
\hline$\Delta$ Anxiety & $-1.02(2.34)$ & $-1.08(2.73)$ & ns \\
\hline$\Delta$ Depression & $-1.45(2.40)$ & $-1.37(2.38)$ & ns \\
\hline \multicolumn{4}{|l|}{ CRDO $(n=51)$} \\
\hline$\Delta$ Dyspnoea & $5.1(6.45)$ & $3.3(4.79)$ & ns \\
\hline$\Delta$ Mastery & 3.3 (3.92) & $1.6(4.10)$ & ns \\
\hline$\Delta$ Emotion & $4.9(6.21)$ & $3.1(5.40)$ & ns \\
\hline$\Delta$ Fatigue & $3.8(5.19)$ & $2.7(4.57)$ & ns \\
\hline Pre-exercise $\mathrm{SaO}_{2}$ & $93.9(3.3)$ & $94.9(2.8)$ & ns \\
\hline $\begin{array}{l}\Delta \mathrm{SaO}_{2} \text { during baseline ISWT } \\
(\mathrm{n}=51)\end{array}$ & $-2.7(4.6)$ & $-7.1(6.75)$ & $<0.0001$ \\
\hline
\end{tabular}

Results are presented as group means (SD). ISWT = incremental shuttle walk test, EWST = endurance shuttle walk test, $\mathrm{HAD}=$ hospital anxiety and depression questionnaire and CRDO = chronic respiratory disease questionnaire.

\section{S111 UNDERSTANDING REASONS FOR PATIENT ATTENDANCE AND NON-ATTENDANCE IN PULMONARY REHABILITATION AND COPD SELF-MANAGEMENT PROGRAMIMES. A QUALITATIVE SYNTHESIS AND APPLICATION OF THEORY}

doi:10.1136/thoraxjnl-2012-202678.116

R Sohanpal, EA Steed, SJC Taylor. Barts and The London School of Medicine and Dentistry, Queen Mary University of London, London, UK

Introduction and Objectives Reviews have suggested that demographic and clinical factors are insufficient to understand poor attendance at interventions that promote self-management. An adapted version of the Attitude-Social Influence-Self-efficacy
(ASE) health behaviour model has been used previously to explain participation in asthma self-management (Lemaigre, 2005). In this model, 'attitude' refers to the sum of positive and negative beliefs and evaluation of the behaviour; 'social influence' refers to the perceived social pressure an individual may feel to perform a particular behaviour; and 'external barriers' are structural or physical barriers.

A systematic review of qualitative studies looking at factors influencing attendance, non-attendance and non-completion in pulmonary rehabilitation (PR) and self-management (SM) programmes amongst patients with chronic obstructive pulmonary disease (COPD) was conducted, and we examined if the adapted ASE model might explain participation behaviour.

Methods We searched eight electronic databases including MEDLINE (1984-2011). Thematic framework synthesis identified emergent themes and sub-themes which were then mapped, where applicable, onto each construct of the adapted ASE model.

Results Six studies were identified, PR $(n=5), S M(n=1)$. Three main themes, 'reasons for attending', 'reasons for not attending' and 'reasons for dropping out' and 33 sub-themes (including psychological, social themes and practical barriers) emerged following data synthesis. Participants' reasons for attending mainly related to improving health or increasing sense of control, whilst reasons for not attending were commonly structural barriers perceived as difficult to overcome. Advice from health care professionals on whether the programme may or may not be beneficial was influential on attendance. Drop out was commonly explained by not seeing improvement in health.

Overall the subthemes identified under 'reasons for attending' and 'reasons for dropping out' commonly mapped onto the 'attitude' construct of the adapted ASE model (see Figure) whilst subthemes identified under 'reasons for not attending' more commonly mapped onto the 'external barriers' construct.

Conclusion Patients attitudes and structural barriers are important in explaining participation behaviour in PR and SM interventions and hence theory based interventions directed at these have potential to improve COPD outcomes.

Abstract S111 Figure 1 Figure Example of a few mapped subthemes onto constructs of the adapted ASE model that explain 'Reasons for attending' 